\title{
Automatic painted surface inspection and defect detection
}

Dipl.-Ing. Hannes Loferer

Micro-Epsilon Messtechnik

Königbacher Str. 15, 94496 Ortenburg

Buying a new car is a highly emotional act: a potential buyer will only spend a significant amount of money if he or she firmly believes in the value of the product under consideration. In this respect, the external appearance of a vehicle is of outmost importance: It is what generates the first impression which will be hard to change by any arguments heard in the ensuing negotiations. Now, imagine a vehicle costing several tens of thousands of Euros, and the first thing you see is an ugly inclusion in the otherwise nice and shiny surface: At a minimum, you will be hesitant to pay the full price asked. Maybe you even walk away from the deal. This is quite commonplace, even with today's optimized painting strategies.

In order to avoid this situation, car manufacturers spend a significant amount of time and energy to visually check the surface of every car very carefully for even minute defects. Special light tunnels are used to generate lighting conditions that facilitate detection of defects down to a few tenths of $\mathrm{mm}$ in diameter. But even with all the technical aids, the visual inspection is never very reliable. It is a widely known fact that detection rates vary with the time of day and are highly affected by human factors such as fatigue and lack of concentration.

The German sensor specialist Micro-Epsilon solves this problem with their revolutionary "reflectCONTROL". Here, the visual inspection is replaced by a reliable and reproducible automated inspection, albeit working in a similar manner to what happens in a light tunnel: Based on the principle of deflectometry, the surface of the vehicle is used as a mirror. Defects cause distortions in the mirrored images that are automatically detected and classified. As an option, found defects can even be automatically marked on the vehicle, simplifying the rework process significantly.

By reliably finding defects right after the paint booth, reflectCONTROL significantly reduces the number of defects that pass into subsequent process steps. With costs for removing defects increasing almost exponentially the farther downstream a defect is found, reflectCONTROL offers a savings potential of millions of Euros per year.

\section{Principle of deflectrometry}

In contrast to traditional vision approaches where the surface is illuminated by a point light source and diffusively reflected light is recorded by a camera, deflectometry uses an area light source and records the specularly reflected light. First of all, this approach allows the system to be used on highly glossy surfaces like the painted exterior of a vehicle where all other approaches using diffusive reflection will fail. Furthermore, it also makes the system extremely sensitive to changes in the curvature of the surface. And this is what allows detection of defects with a height or depth of just a few micrometers. 
But deflectometry comes in multiple flavours: The key to the robust detection offered by reflectCONTROL lies within the employed multi-image approach: for every patch of the surface, multiple images are taken with a sinusoidal stripe pattern shifted into multiple positions, which then is rotated by $90^{\circ}$ and shifted once more. This elaborate data acquisition scheme guarantees a perfectly uniform sensitivity across the whole surface, ensuring that every defect shows up clearly in the recorded images no matter where it is on the surface and how it is oriented. Furthermore, unavoidable statistical fluctuations in the individual images have no chance to be mistaken for pseudo-defects as they cancel each other out when combining the images.

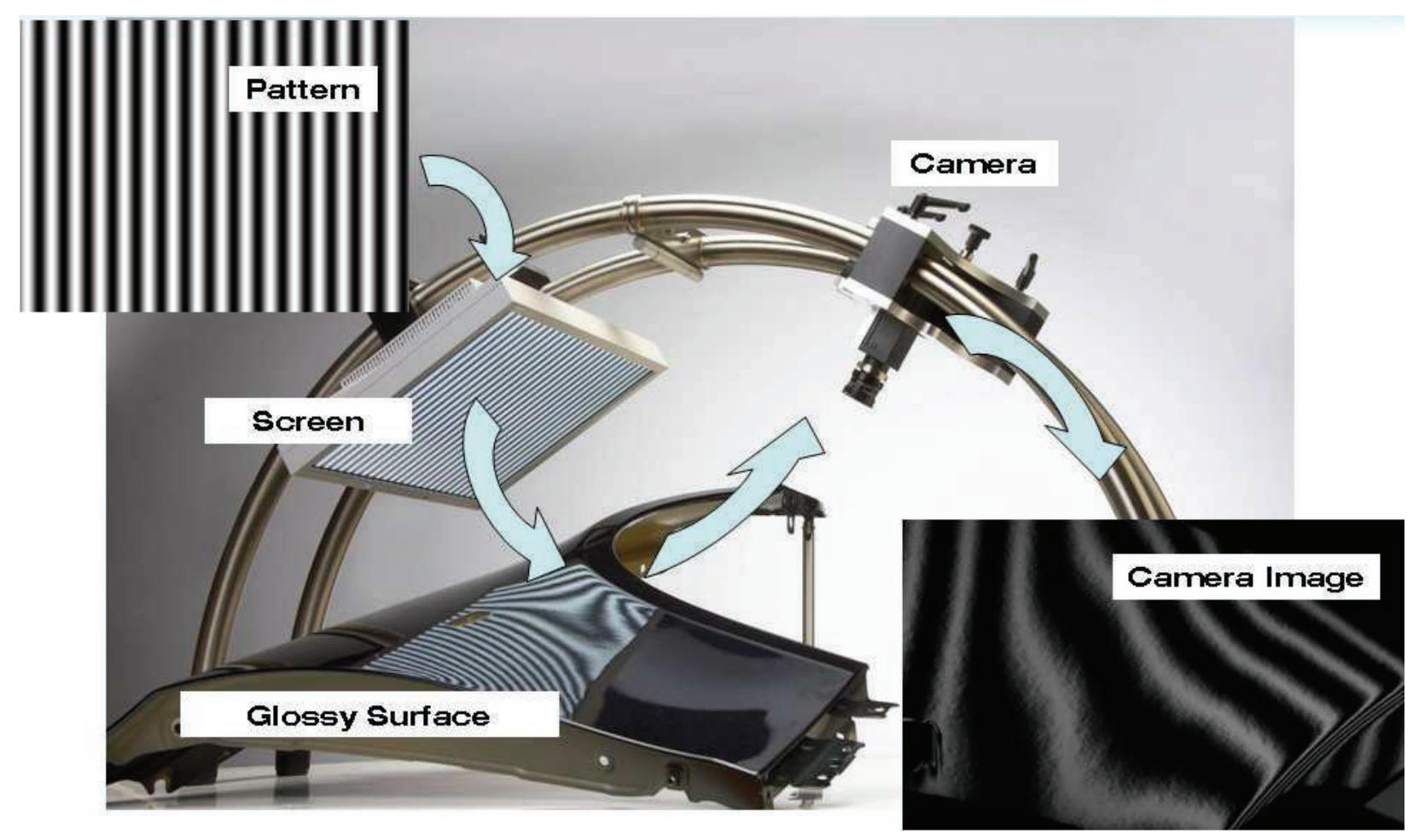

By adding sophisticated image processing algorithms, reflectCONTROL will reliably detect all relevant defects typically found on any vehicle, such as: contacts, inclusions/bulges, overpainted fluff / hair, adhesive residues, mottles, craters, paint delamination, paint drops, runs, pinholes, overspray, moulding defects, scoring, shell defects, slide marks, welding beads, spitters, specks, partial / missing paint, soiling and water droplets.

\section{Inspecting Vehicles}

The inspection of the complete exterior of a vehicle at typical production rates of about 40-60 units per hour in order to check for defects as small as $0.3 \mathrm{~mm}$ in diameter might appear a herculean task. In fact, it is. To accomplish this feat, reflectCONTROL employs 4 robots working in parallel, each one of them equipped with a sensor consisting of one large monitor and 4 cameras. Every camera takes 8 images per inspection position, each inspection taking less than $1 \mathrm{~s}$. With the robot moving at comfortable speeds, 30 positions can be inspected within 60s cycle time. Using 2 MegaPixel cameras, this totals in almost 7 billion gray-values that need to be recorded and processed for every single vehicle. This can only be done with optimized software working in a distributed system. 


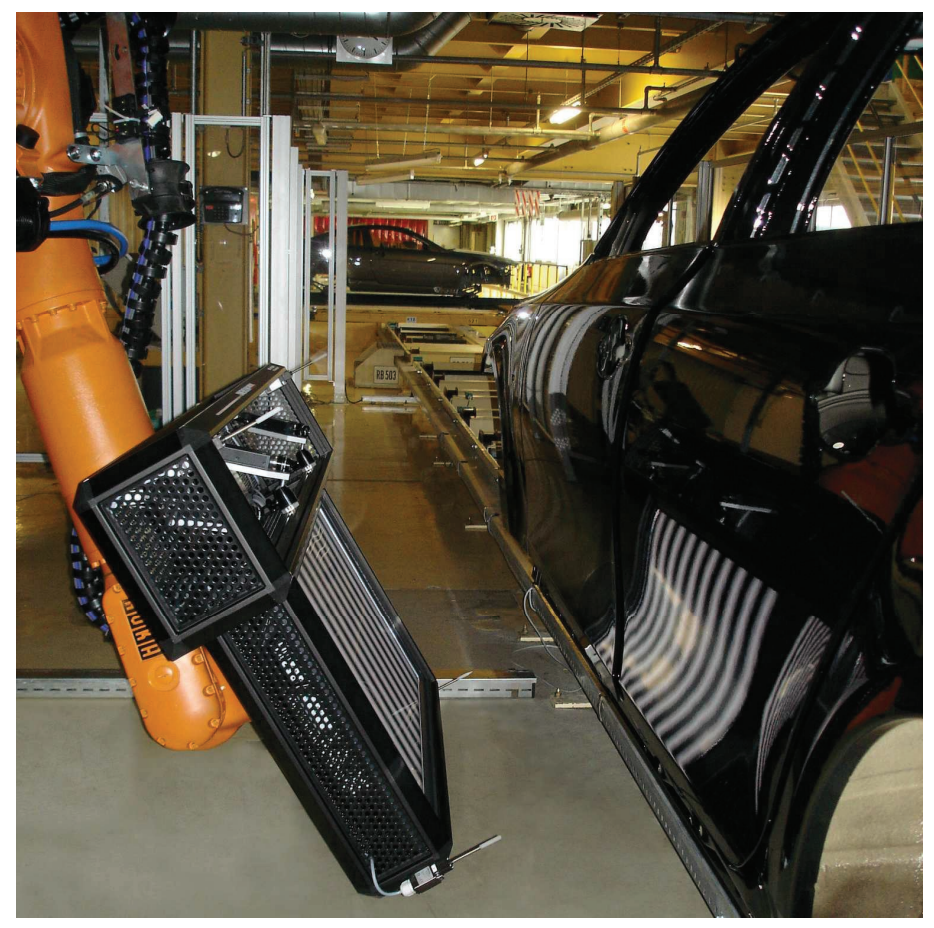

\section{Solutions from the sensor specialists}

Micro-Epsilon is a renowned and trusted source for (almost) any kind of distance and dimensional measurements. Many years of experience has led to a consistent portfolio of specialized and highly accurate sensors. The systems division of Micro-Epsilon takes this success story one step further: By adding mechanics and software to the actual sensors, highly capable systems are created to meet and quite often exceed application requirements. All parts of the system are created by one homogeneous team of experts in constant communication with each other, resulting in reliable systems you can trust.

\section{Proven solution}

Together with a major European car manufacturer and the University of Passau, Germany, extensive studies on more than 1,000 vehicles were performed using a test system integrated into the production flow. Here it showed that performance levels achieved by reflectCONTROL are by far superior to those of visual inspection. Based on this extremely positive outcome, the manufacturer now wants to take the next step: integration of a full system into the line to make sure it can keep up with the requirements of an actual inline system. Micro-Epsilon is looking forward to this challenge. 